\title{
Palmeras aceiteras del Ecuador: estado del arte en la investigación de nuevos recursos oleaginosos provenientes del bosque tropical
}

\author{
Rommel Montúfar-Galárraga ${ }^{1}$ \& Grischa Brokamp ${ }^{2}$ \\ ${ }^{1}$ Pontificia Universidad Católica del Ecuador, Escuela de Ciencias Biológicas, Laboratorio de \\ Ecología y Genética, Quito, Ecuador. \\ rjmontufar@gmail.com \\ ${ }^{2}$ Institut für Systematische Botanik und Pflanzengeographie, Freie Universität, Altensteinstr. 6, \\ D-14195 Berlin, Alemania.
}

Recibido: 19, 06, 2011; aprobado: 15, 08, 2011

RESUMEN.- Ecuador tiene una elevada diversidad de palmeras (Arecaceae) en su territorio (134 especies nativas), algunas de las cuales constituyen potenciales fuentes de aceites. Se realizó una revisión bibliográfica que incluyó 24 trabajos que reportan la composición de ácidos grasos y contenido de aceites de frutos de palmeras pertenecientes a los géneros Astrocaryum, Attalea, Bactris, Cocos, Elaeis, Euterpe, Mauritia, Oenocarpus, y Phytelephas. En base a los análisis de PCA y UPGMA, se observa que los aceites derivados de palmeras pueden clasificarse primariamente en función del tejido de aislamiento: (a) aceites derivados de mesocarpo ricos en ácidos grasos mono-insaturados (MUFAs), y (b) aceites derivados del endospermo ricos en ácidos grasos saturados (SFAs). En particular, el mesocarpo de Phytelephas aequatorialis y el endospermo de Mauritia flexuosa tienen una elevada proporción de ácidos poli-insaturados (PUFAs). A pesar del potencial de los aceites derivados de frutos de palmeras como nuevas fuentes oleaginosas; limitaciones en la productividad de aceite, en el manejo, extractivismo de especies dioicas, y la conservación de los recursos fitogenéticos requieren ser estudiados. PALABRAS CLAVE: palmeras aceiteras, oleaginosas, aceites, Arecaceae, Ecuador.

ABSTRACT.- Ecuador has one of the highest palm's population diversity in the Neotropic (134 natives species); some of them have the potential like new sources of oils and fats. A bibliographic search showed 24 studies describing fatty acid composition for Ecuadorian palms (Astrocaryum, Attalea, Bactris, Cocos, Elaeis, 
Euterpe, Mauritia, Oenocarpus, and Phytelephas). PCA and UPGMA analysis showed that oils derived from palm fruits can be classified in function of tissue from where it was isolated: (a) oils derived from the mesocarp with high concentration of MUFAs, and (b) oils derived from the endosperm with high concentration of SFAs. Oils derived from Phytelephas aequatorialis (mesocarp) and Mauritia flexuosa (endosperm) showed a peculiar concentration of PUFAs. Despite of the economic potential of native palms like new sources of edible and industrial oils, the knowledge of the oil productivity, management of natural populations, and the conservation of the genetic resources need to be studied.

KEYWORDS: Oil palms, oleaginous, oils, Arecaceae, Ecuador.

\section{INTRODUCCIÓN}

La población humana requiere de nuevas y más productivas fuentes de aceites, tanto para el consumo humano, la producción de productos cosméticos y la generación de biocombustibles. La demanda de aceites a nivel global aumentó significativamente desde 1961 hasta la actualidad. Por ejemplo, la producción de aceite de palma (Elaeis guineensis) y de soya (Glycine max) se incrementó en un $51.3 \%$ y $42.8 \%$, respectivamente, durante el período 19612003 (1). Para el año 2011, se calcula una demanda global de aceite de palma de 46.6 millones de toneladas métricas; si las tasas de consumo se mantienen, la demanda de aceites de palma para los próximos años podría quedar insatisfecha. Esta dinámica del mercado de aceites está influenciada por dos factores: (a) una demanda creciente de aceites para la generación de biocombustibles y cosméticos; y (b) una demanda más exigente para la generación de aceites comestibles y beneficiosos para la salud humana.

El ácido graso (fatty acid, FA) es la unidad elemental de las grasas y aceites. Dependiendo del grado de saturación de sus cadenas de carbono, los ácidos grasos son clasificados en saturados (SFAs), mono-insaturados (MUFAs) y poli-insaturados (PUFAs; 2). Los aceites de origen vegetal pueden ser ricos en SFAs (Cocos nucifera 92.6\%, Elaeis guineensis-semilla $81.9 \%$, Theobroma cacao $62.9 \%$, Mangifer indica $52.8 \%$; 2) y en MUFAs (Oenocarpus bataua $80 \%$, Mauritia flexuosa $81.3 \%$, Olea europaea 75\%, Corylus avellana $83.1 \%$, Macadamia tetraphylla $80.4 \%$, Persea americana $67.8 \%$, Brassica alba 68.5\%; 2, 3, 4). Algunos aceites vegetales también poseen altos porcentajes $(\sim 40 \%)$ de PUFAs (Phytelephas aequatorialis 21-35\%, Mauritia flexuosa $17 \%$, Brassica napus 31.5\%, Prunus armeniaca $28.8 \%$, Avena sativa $38.9 \%$, 
Acer saccharum 40.6\%; 2, 4, 5). En cambio, las grasas de origen animal son generalmente ricas fuentes de SFAs; a excepción de los aceites derivados de peces los cuales tienen altas concentraciones de PUFAs (1).

El consumo de ácidos grasos (aceites y grasas) es un componente importante en la dieta. El ser humano consume aproximadamente $25 \mathrm{~kg}$ de aceites y grasas por persona/año, mayormente $(80 \%)$ de origen vegetal (6). El tipo de ácido graso ingerido en la dieta (PUFAs, MUFAs, SFAs) juega un rol importante en la salud humana. Una dieta con altas proporciones de SFAs está asociada a la incidencia de enfermedades coronarias, hipertensión y elevadas tasas de colesterol $(1,7)$. Por ejemplo, aceites con altas concentraciones de ácidos grasos saturados como el láurico (C12:0), mirístico (C14:0) y palmítico (C16:0) incrementan el colesterol LDL (lipoproteinas de baja densidad; 1); mientras que, el reemplazamiento de SFAs (C12:0-C16:0) por MUFAs reducen las concentraciones de colesterol LDL (1). Adicionalmente, existe una creciente evidencia que sugiere que la ingesta de PUFAs reduce la incidencia de riesgo por enfermedades coronarias (1); y en particular se ha demostrado que el consumo de PUFAs como el ácido araquidónico (serie omega 6) y el eicosapentaenoico (serie omega 3) son elementos indispensables para el metabolismo humano (6). En base a la evidencia disponible de la relación entre salud y consumo de aceites, la Organización Mundial de la Salud (OMS) ha sugerido que la ingesta de ácidos grasos saturados no debería aportar más del 10 $\%$ de la energía requerida por un individuo adulto (8).

Las correlaciones entre el tipo de aceite y salud son todavía tentativas debido a la multiplicidad de factores involucrados en el metabolismo de las grasas (estilo de vida, base genética, hábitos alimenticios). A pesar de ello, existe un claro consenso en relación al tipo de aceite ideal para la dieta humana; en particular aceites ricos en MUFAs como el aceite de oliva, y aceites ricos en PUFAs como los derivados de peces son sugeridos como fuentes saludables de aceites comestibles (2). Adicional, a la importancia de los ácidos grasos a la dieta humana, los aceites son fuentes importante de provitaminas liposolubles (carotenos, tocoferoles, tocotrienoles, etc.).

La ausencia de una equilibrada y variada ingesta de ácidos grasos y vitaminas en la dieta contribuye, parcialmente, a las altas tasas de desnutrición latentes en el país; por ejemplo el 26 $\%$ de los niños ecuatorianos menores a cinco años tienen desnutrición crónica; y de estos, el $60 \%$ habitan en áreas rurales (9). Las grasas son relativamente costosas, por lo cual la alimentación de las personas más pobres, en particular en el medio rural, disponen de poca grasa ó de grasas no saludables ricas en 
SFAs (8). En Ecuador, el consumo de grasas saturadas de origen animal (cerdo, res, etc.) está ampliamente extendido en la alimentación en detrimento de una ingesta de aceites de origen vegetal saludables y variados en composición de ácidos grasos. A partir de 1970, con el desarrollo de la industria aceitera ecuatoriana, el consumo de grasas de origen animal fue suplantado, parcialmente, por aceites vegetales derivados de la palma africana, con altos porcentajes de SFAs (48-50\%). A pesar de las recomendaciones de la FAO/OMS para la reducción de SFAs en la dieta humana, Ecuador lidera el consumo el aceite de palma en América Latina con 233.15 kcal/persona/día, seguido de Colombia (141.43 kcal/persona/día) y Venezuela (71.81 kcal/persona/día; 10).

El objetivo del presente trabajo es aportar al conocimiento actual sobre el potencial de las palmeras americanas (en particular las palmeras ecuatorianas) como nuevas fuentes de aceites y grasas, su diversidad bioquímica y las limitaciones que implica su explotación comercial. Se proporciona una revisión de las especies que fueron históricamente utilizadas para la obtención de aceites vegetales en Ecuador. El mercado mundial de aceites se ha caracterizado por la explotación comercial de un reducido número especies oleaginosas. Nuevas fuentes de aceites vegetales permanecen subvaloradas en nuestros bosques tropicales.
Palmeras como fuentes de aceites vegetales provenientes de los bosques tropicales. Ecuador posee una alta diversidad de palmeras (Arecaceae) con 134 especies distribuidas en el litoral, estribaciones andinas y Amazonía (11). Los frutos de las palmeras (mesocarpo y endospermo) sintetizan una gran diversidad de ácidos grasos, propiedad metabólica que ha sido aprovechada por el ser humano para el uso, explotación y comercialización de aceites vegetales.

Los frutos de palmeras nativas del Ecuador son reportados por la literatura etno-botánica como fuentes de aceites para los pueblos amerindios y colonos $(12,13,14,15,16,17)$. Las palmeras Attalea colenda, Oenocarpus bataua, Bactris gasipaes, Elaeis oleifera, Mauritia flexuosa son mencionadas en la literatura como especies que presentan un elevado potencial para la explotación de los aceites derivados de los frutos (12, 16, 18). Attalea colenda, localmente conocida como palma real, es una palmera silvestre, monoica, seudo-endémica del litoral de Ecuador y sur occidente de Colombia, con un rango altitudinal entre los 0 a 500 m.s.n.m. (19). En Ecuador, esta palmera esta presente en los bosques húmedos de la provincia de Esmeraldas y en los bosques secos estacionales de Manabí. Poblaciones extraordinarias de A. colenda sobre los 1000 m.s.n.m son reportados en la provincia de El Oro, Bolívar y Santo Domingo (20). Esta palmera tiene tallos superiores a los $35 \mathrm{~m}$ 
de altura y diámetros entre 35 a $60 \mathrm{~cm}$; con infructescencias de más de $1.5 \mathrm{~m}$ de largo con abundantes frutos de 4 a $6 \mathrm{~cm}$ de largo portadores de un endospermo rico en aceites de tipo láurico $(47.2 \%)$ y ácido oleico $(13.5 \% ; 21,22,23)$.

Antes del establecimiento de plantaciones comerciales de palma africana (posterior a 1960), la incipiente industria aceitera ecuatoriana explotó comercialmente aceites provenientes de frutos (mesocarpo y endospermo) de palmas silvestres como $A$. colenda. El botánico Misael Acosta Solís describe ya en 1960 (22) la comercialización de la almendra seca (endospermo) de $A$. colenda por las comunidades negras del noroccidente ecuatoriano para la extracción de aceite. Las primeras referencias formales sobre la industrialización del aceite de $A$. colenda datan de 1987 por la empresa Ales, ubicada en las cercanías de la ciudad de Manta (21). Acosta-Solís (22) menciona que Ecuador exportó a Colombia y Estados Unidos más de 1.608.300 kilos de semillas o almendras de la palma real en un solo año (presumiblemente cerca de 1960). En la actualidad, los frutos de $A$. colenda no poseen importancia económica a pesar que fueron históricamente un ítem de exportación para el país (20).

A nivel de productividad, los frutos de $A$. colenda constituyen una alternativa promisoria para la producción de aceites. Estudios realizados en las provincias de Manabí y Esmeraldas, revelan que una hectárea de bosques secundarios o bajo plantaciones agroforestales, con una población de 50 individuos sexualmente reproductivos de palma real pueden generar en promedio 900 kilogramos de aceite por año (23). Considerando que $A$. colenda se adapta bien en ecosistemas secos en donde plantaciones de palma africana no se adaptaría, la explotación de $A$. colenda podría ser una actividad económicamente rentable y una importante fuente de ingreso monetario a las comunidades locales.

El género de palmeras Attalea tiene otras especies con potencial oleaginoso para Ecuador. A. butyracea es una palmera neotropical presente en los bosques húmedos tropicales (bosques aluviales) de la región septentrional de la Amazonía Ecuatoriana (19). En esta región, los frutos de A. butyracea son utilizados raramente como fuentes de aceites por las etnias Siona, Cofán, Kichwa, Secoya y Wao (12); no existen comercialización de frutos ni del aceite de Attalea en los mercados amazónicos de Ecuador. En Colombia, A. butyracea es la palmera con mayor número de usos en Colombia y la extracción de aceites comestibles de sus frutos persiste ocasionalmente hasta hoy día (24). Las semillas de A. butyracea contienen 50 a $68.5 \%$ de un aceite semi-sólido y de color blanco, parecido al aceite del cocotero (24); con una mayoritaria fracción de ácido láurico (42.9 $\%$; 25). En los bosques amazónicos de tierra firme de la amazonía ecuatoriana, 
la palmera $A$. maripa ha sido reportada con similares características oleaginosas. El mesocarpo de A. maripa posee entre 13 a $32 \%$ de contenido de aceites, y en particular el ácido oleico es la fracción más representativa (40 a $43 \%$ ), seguido de ácido mirístico (17.9 a 19.5 \%) y ácido palmítico (17.9 a $19.3 \%$; 26, 27); mientras que el endospermo (kernel) es mayoritariamente láurico (26, 28). Un ejemplo del potencial económico de este género de palmeras constituye la especie $A$. phalerata, presente en el bosque amazónico de Perú, Bolivia y Brasil (29). En los años 1980, aproximadamente 250 mil toneladas métricas de semillas de $A$. phalerata fueron procesadas para la extracción de aceites en Brasil (30). La extracción artesanal de aceites de sus frutos, para fines alimenticios y bio-combustibles, es una fuente de ingresos para cientos de miles de campesinos y productores rurales (31).

Los frutos de la palmera Oenocarpus bataua -conocida localmente como ungurahua, ungurahui, chapil, seje, mil pesos- constituye una importante fuente sub-valorada de aceites comestibles. $O$. bataua es una especie de amplia distribución en las regiones tropicales de América del sur (0 a 1.200 m.s.n.m.) y la isla de Trinidad en el Caribe. Es una palmera silvestre y monoica, con un tallo superior a los $20 \mathrm{~m}$ de alto y un diámetro entre 20 a $40 \mathrm{~cm}$ (19). En Ecuador, esta palmera esta presente en los bosques húmedos tropicales y subtropicales (hasta los 1.200 m.s.n.m.) a ambos lados de la cordillera. O. bataua es la especie más citada en la literatura etnobotánica como fuente de aceites para consumo humano $(17,22,32,33,34,35)$. El aceite es extraído del mesocarpo (pulpa) a través de un proceso de cocción, maceración, y filtración por los pueblos amazónicos. Hoy en día, el consumo de aceite de ungurahua tiende a desaparecer en la región. En 1974, Calvante (22) reporta la desaparición del aceite de ungurahua del mercado de Belém (Brasil); una situación similar se observa en los poblados de la amazonía de Ecuador y Perú. La venta de frutos para la elaboración de bebidas no alcohólicas (leche de seje, jugos) derivado de la pulpa permanece como una actividad económicamente marginal y de subsistencia de las etnias y colonos en la Amazonía.

Oenocarpus bataua fue clasificada por la FAO entre las especies con potencial para la domesticación (18). La principal característica química del mesocarpo de los frutos de O. batana es la predominancia de ácidos grasos insaturados $(33,36)$. La composición química de este aceite es similar al aceite de oliva; con una alta concentración de ácido graso oleico $(>73 \%)$; una baja proporción de ácidos grasos saturados y la presencia de ácidos grasos poli-insaturados (36). La fracción de esteroles esta mayormente representada por $\Delta 5$-avenasterol, un potencial antioxidante. Adicionalmente, el aceite tiene una concentración de 
beta-caroteno (precursor de la vitamina A) superior a otras fuentes de aceites vegetales como el girasol o la soya, pero remarcablemente baja en relación a las concentraciones del aceite derivado de la palma africana (36); mientras que posee una alta concentración de $\alpha$-tocoferoles o vitamina $\mathrm{E}(1.704 \mathrm{mg} / \mathrm{kg})$. Estas propiedades químicas definen al aceite de ungurahua (mesocarpo) como un aceite oleico de alta calidad para el consumo humano (36). En cambio, la semilla de la ungurahua (endospermo) es rico en aceites láuricos $(42 \%)$ y oleico $(16 \%)$, y podría ser explotado simultáneamente (37).

Referencias históricas sobre la explotación comercial de los frutos de la ungurahua son escasas. Brasil explotó comercialmente las poblaciones silvestres de $O$. bataua para la extracción del aceite durante la primera mitad del siglo XX. Para el año 1940, la exportación de aceite de ungurahua estuvo cerca de las 100 toneladas (35). Después de la segunda guerra mundial, la exportación de aceite de ungurahua declinó dramáticamente hasta su desaparición. La sobreexplotación de las poblaciones silvestres próximas a centros urbanos y la competencia con la industria aceitera de la oliva europea influyeron en la desaparición de esta actividad económica (35). Acosta-Solís (22) describe la exportación de grandes cantidades de frutos de $O$. bataua desde Ecuador a Colombia, y la venta de estos frutos a las fábricas aceiteras de Manta y Guayaquil durante la segunda guerra mundial. En
Colombia, se reporta la exportación de aceite de ungurahua a Francia durante la década de los noventa (38). Actualmente, en las ciudades amazónicas de Iquitos, Cayena, Belém, Manaos, entre otras, se comercializa la pulpa de ungurahua para la fabricación artesanal e industrial de helados y repostería. En la Amazonía occidental, iniciativas locales extraen el aceite de ungurahua para fines cosméticos (jabones, aceites corporales, champú) y como tónico capilar en menor escala.

Oenocarpus bataua es una palmera silvestre abundante en la tierra firme del bosque amazónico. Con una densidad promedio de 35 palmas reproductivas por hectárea en la región de tierra firme de Cuyabeno, se proyecta una producción de $51.8 \mathrm{~kg}$ de aceite/hectárea/bianual. Estos datos reflejan un potencial extractivista del aceite de ungurahua proveniente de poblaciones naturales (39). Un manejo técnico de las poblaciones silvestres constituye una opción para la explotación del recurso. En particular, para una región que sufre de desnutrición, con un déficit proteico y alta tasas de consumo de ácidos grasos saturados, la producción y consumo de aceite de ungurahua es recomendable, y susceptible a generar una actividad económica importante.

Bactris gasipaes, localmente conocida como chontaduro o pijuayo, es la única especie de palmera domesticada en el neotrópico. Es una palmera cespitosa, monoica, de tallo mediano (hasta 
15 a $20 \mathrm{~m}$ ) que crece abundante en regiones tropicales y subtropicales húmedas del Ecuador. El fruto del chontaduro es utilizado por los pueblos amazónicos como fuente de carbohidratos (hasta 79 $\%$ del peso seco), proteínas (hasta 11.3 $\%$ del peso seco) y aceites (mayormente oleico, hasta $29.4 \%$ del peso seco; 40 ). Los frutos se consumen directamente (previamente cocinados), o se fabrica la chicha y harina de chontaduro. Los frutos y derivados son comercializados en las ciudades tropicales de tierras bajas de Ecuador y América del Sur. B. gasipaes produce dos productos con importancia económica: el palmito (hoja joven o cogollo) y el fruto (pulpa o mesocarpo). La industria del palmito en Ecuador produce más de 27 mil toneladas (año 2008) de palmito en conserva, llegando a convertir a Ecuador en el primer productor mundial de palmito (año 2008; 41), y representando el $1 \%$ de las exportaciones no petroleras para el período 2004-2008 (año 2008; 41). A pesar del impulso de la agroindustria del palmito de $B$. gasipaes, poco o nada de interés comercial ha sido proporcionado al fruto de la misma especie. El cultivo del fruto se realiza a nivel de subsistencia por los grupos amerindios y son escasas las iniciativas para explotar los aceites derivados del fruto. La domesticación de B. gasipaes en el neotrópico aparentemente remonta desde tiempo prehistóricos (42); siendo el objetivo de este proceso el mejorar las características volumétricas y nutritivas del fruto. Existe una amplia diversidad genética generada en el transcurso del proceso de domesticación del fruto en la América tropical $(40,43)$; sin embargo, la destrucción de los bosques y la introducción de variedades comerciales para explotación de palmito favorecen la erosión y contaminación de la integridad genética de las variedades locales vía hibridación introgresiva $(43,44)$. Elaeis oleifera es la pariente americana de Elaeis guineensis (palma africana). Esta palmera está presente en las regiones tropicales de Centro y Sur América; en Ecuador se conocen poblaciones de esta especie en las regiones tropicales húmedas de las provincias de Pastaza y Morona-Santiago (19). Al ser una especie filogenéticamente próxima de $E$. guineensis, ha sido incorporada dentro de los programas de mejoramiento genético de la palma africana y en particular en la generación de híbridos inter-específicos (45). E. oleifera es todavía un cultivo económicamente no viable debido a la baja productividad de aceites (comparado con E. guineensis), sin embargo posee una importante potencial debido a que ofrece un aceite de alta calidad, baja altura del individuo y resistencia a enfermedades (46).

Mauritia flexuosa, localmente conocida como buruti, morete, aguaje, cananguchal, es una palmera de creciente interés económico en la región. Es una palmera monoica, monocaule y arborescente con tallos superiores a los 25 
metros de alto y diámetro entre 30 a 60 centímetros (19). M. flexuosa forma densas poblaciones monoespecíficas (hasta 246 troncos por hectárea) en los bosques amazónicos sobre suelos mal drenados (pantanos y planicies aluviales). El mesocarpo de sus frutos es aceitoso y con un alto contenido de vitamina A. Los usos alimenticios de los frutos de M. flexuosa, como la extracción de la pulpa para helados y repostería o el consumo directo de sus frutos, prevalecen sobre su potencial como fuente oleaginosa.

Los frutos de E. oleracea tienen un importante potencial oleaginoso. En Ecuador, las poblaciones de E. oleracea están restringidas al estuario del río Santiago y Cayapas (Esmeraldas). Es una palmera arborescente, monoica y cespitosa, cuyas poblaciones naturales fueron negativamente afectadas por la cosecha de su palmito alrededor de la década de los 70. Actualmente, se comercializa en el mercado internacional (Europa y Estados Unidos) productos procesados derivados de los frutos de E. oleracea (asaí) como energizantes y bebidas vitamínicas. Al igual que M. flexuosa, los productos procesados de E. oleracea con fines comestibles prevalecen sobre su potencial oleaginoso.

Diversidad de ácidos grasos en la flora de palmeras ecuatoriana. Se realizó una revisión bibliográfica sobre la composición química de los aceites derivados del mesocarpo y endospermo de palmeras oleaginosas. En particular, se enfatizó la búsqueda de información bibliográfica para especies de palmeras reportadas en el territorio ecuatoriano. Se incluyeron 24 trabajos que reportan datos sobre la composición química del aceite derivado de frutos de 12 especies de palmeras, dentro de los géneros Oenocarpus, Bactris, Mauritia, Elaeis, Astrocaryum, Attalea, Cocos, Phytelephas, Euterpe; y de las especies comerciales Olea europaea (oliva) y Elaeis guineensis (palma africana). Para varias especies se obtuvo información bioquímica del mesocarpo (m) y del endospermo/ kernel (e), lo cual incrementó el número de muestras a 19 (tabla 1). Solamente los datos de Phytelephas aequatorialis y Attalea colenda provienen de poblaciones ecuatorianas. Los valores (porcentajes) máximos y mínimos para los ácidos grasos registrados y el contenido de aceite del mesocarpo o endospermo son reportados en la tabla 1. Con la finalidad de analizar la variabilidad de ácidos grasos en palmeras se realizó un análisis de componentes principales (PCA) y un UPGMA (Unweighted Pair Group Method with Arithmetic Mean) utilizando los valores medios obtenidos en la revisión bibliográfica para cada ácido graso en cada especie. Estos análisis fueron realizados con los seis ácidos grasos más abundantes en las especies estudiadas: láurico, mirístico, palmítico, esteárico, oleico y linoleico. Ácidos grasos con reducida representación en la muestra fueron excluidos del análisis 
(caprílico, cáprico, palmitoleico, alfa linolénico) con la finalidad de reducir la presencia de ceros en la matriz. El PCA fue llevado a cabo con la matriz de correlaciones que incluyó un set de seis ácidos grasos como descriptores y 19 datos de especímenes como objetos. Para el análisis de UPGMA la matriz de 6 descriptores x 19 objetos fueron estandarizados (Z-scores) y transformados a una matriz de distancia euclidiana. Los análisis estadísticos fueron llevados a cabo con el programa R-Package (47). Los resultados del PCA y UPGMA son ilustrados en la figuras 1 y 2 .

Los aceites derivados de palmeras tienen una amplia variabilidad bioquímica (tabla 1). La variabilidad observada puede ser explicada por varios factores: (i) Las palmeras oleaginosas tienen amplios rangos de distribución biogeográfica; y consecuentemente una alta variabilidad fenotípica. La mayoría de los trabajos incluidos en el presente estudio reportan resultados de poblaciones locales; pocos trabajos incluyen un muestreo sistemático a nivel regional con varias poblaciones a lo largo del rango de distribución. (ii) Variaciones en los métodos de extracción de lípidos y en las técnicas cromatográficas para la detección de ácidos grasos. (iii) Diferentes métodos de colecta, transporte y almacenamiento de frutos y tejidos vegetales. (iv) Diferentes diseños experimentales para el muestreo de frutos en el campo y en las réplicas de laboratorio. $(v)$ Inadecuado conocimiento de la taxonomía de palmeras.
El primer componente del PCA separa los aceites de palmeras en función del tipo de tejido: endospermo y mesocarpo. Los aceites derivados del endospermo están caracterizados por altos porcentajes de SFAs, como el mirístico (C14:0) y láurico (C12:0). En particular, los aceites derivados del endospermo de las especies del género Astrocaryum (>25\%), Attalea maripa (24.6-26.2 \%) y Elaeis oleifera $(25.6 \%)$ tienen altos porcentajes de mirístico. La única muestra derivado del mesocarpo con altos porcentajes de mirístico proviene de A. maripa (17.9$19.5 \%$ ). Mientras que, el endospermo de Bactris gasipaes (60.0\%), Astrocaryum chambira y A. murumuru (50 $\%)$, Elaeis guineensis (46-48 \%), Attalea colenda (47\%) son ricas fuentes de láurico. Aceites ricos en ácidos grasos mirístico y láurico constituyen fuentes importantes de aceites industriales y cosméticos.

Los aceites derivados del mesocarpo son mayormente mono-insaturados (MUFAs), y en preferencia dominados por el ácido oleico. En particular, el mesocarpo de los géneros Oenocarpus (>70 \%), Mauritia (71.6-80\%), Euterpe (49.8-56.2\%) y Bactris (41-50.3\%) son fuentes importantes de ácido oleico. La composición y proporción de ácidos grasos de los géneros anteriormente mencionados es similar a los valores reportados para el aceite de Olea europaea (oliva) . 
Un tercer grupo de aceites esta caracterizado por altos porcentajes de ácidos poli-insaturados (PUFAs). El aceite derivado del endospermo de Mauritia flexuosa posee una composición química diferente a lo observado en otros aceites derivados del endospermo. El aceite del endospermo de $M$. flexuosa posee una alta concentración de linoleico (16.1-36.1\%) y oleico (25-53\%), y porcentajes variables (0.2-13.5\%) para alfa linolénico; y el aceite del mesocarpo de Phytelephas aequatorialis tiene altos porcentajes de linoleico (21-31.9\%) y oleico (15-33.5\%). Similares patrones de agrupamiento reportados por el PCA (figura 1) fueron obtenidos en los análisis de UPGMA (figura 2).

El contenido de aceite (\% en peso seco, tabla 1) es variable entre los frutos analizados. Los endospermos más ricos en aceites provienen de Cocos nucifera (62.4-68 \%), Attalea butyracea (43-68.5 \%) y Astrocaryum chambira (40.8-41.4 $\%)$. Los mesocarpos más ricos en aceites derivan de Bacris gasipaes (72.7 \%) у Oenocarpus bataua (44.9-58.2 \%).

\section{Limitaciones actuales para la explotación de las palmeras silvestres y conservación de los recursos fitogenéticos.}

\section{Los largos ciclos de vida de las pal-} meras.- Las palmeras arborescentes tienen una lenta tasa de crecimiento de tejidos, y consecuente, un desarrollo tardío de estructuras sexuales. Estudios realizados en palmeras arborescentes del género andino Ceroxylon reportan que los individuos de la especie Ceroxylum alpinum tardan 57 años para producir un tallo, y 83 años en iniciar su vida reproductiva (48). La palmera arborescente Attalea colenda requiere de 30 a 40 años para generar un individuo adulto con un tallo promedio de 15 metros de alto y potencialmente reprocutivo (23); y la tagua (Phytelephas aequatorialis) requiere por lo menos de 14 a 15 años para desarrollar estructuras sexuales (22). No existe información del ciclo de vida para otras palmeras tropicales, pero debido a su similar morfología y fisiología entre palmeras arborescentes, se esperaría largos períodos para la formación de tejidos y estructuras sexuales. Los largos periodos para el desarrollo de estructuras sexuales (inflorescencias / infructescencias) son un limitante para el establecimiento de cultivos comerciales; las palmeras silvestres no pueden competir con variedades comerciales de Elaeis guineensis y Cocos nucifera en los cuales la fructificación se desarrolla a los 2 o 3 años de vida del individuo.

La respuesta y adaptación de las palmeras silvestres a sistemas agrícolas ha sido escasamente estudiado; y una parte significativa de la información está disponible como literatura no convencional o no publicada. Balick (25) reporta estudios de plantaciones comerciales de Oenocarpus bataua en Brasil (Embrapa), 
sin embargo los resultados de estas investigaciones no son disponibles. Iniciativas agronómicas en localidades de la Amazonía Peruana reportan individuos de Oenocarpus bauata y Mauritia flexuosa con altas tasas de crecimiento en sistemas agrícolas. Por ejemplo, en las localidades de Jenaro Herrera (Loreto, Perú) y Archidona (Napo, Ecuador) se observa individuos de M. flexuosa sobre pastizales con tallos menores a dos metros de alto y portando varias infructescencias. Similares observaciones fueron reportadas en la Estación Científica Río Palenque (Ecuador), en donde un individuo de Oenocarpus bataua, bajo fertilización temporal y exposición solar completa, produjo inflorescencias a un metro del suelo (49). Las observaciones reportadas anteriormente, aún siendo informales, son un indicio del potencial de manejo de palmeras silvestres en sistemas agrícolas. Para potencializar el uso comercial de palmeras silvestres se requiere de una investigación agronómica dirigida a explorar la capacidad de respuesta de las palmeras a sistemas agrícolas, y la influencia del medio en la composición química del fruto.

Un aspecto importante de la biología de las palmeras es la naturaleza dioica de algunas especies (Mauritia flexuosa, Phytelephas aequatorialis, Aphandra natalia). Una especie es dioica cuando existe una separación física entre individuos con flores masculinas y flores femeninas (portadora de frutos). El establecimiento de plan- taciones comerciales de la palmera $M$. flexuosa ha sido desalentado debido al desconocimiento de los mecanismos que determinan la sexualidad de las semillas, plántulas o juveniles. Para el cultivo de especies dioicas es prioritario establecer una relación óptima entre individuos masculinos y femeninos que favorezca la polinización y la posterior productividad de la plantación. Para las especies dioicas con potencial oleaginoso es prioritario la investigación para el desarrollo de marcadores anatómicos y genéticos que facilite la identificación temprana de la sexualidad de las semillas y plántulas.

Incremento en la productividad de aceites de los frutos de palmeras silvestres.- Los frutos de Oenocarpus bataua, y de otras especies de palmeras, poseen un alto porcentaje de aceite en el mesocarpo; pero un relativamente bajo porcentaje de mesocarpo en relación al tamaño del fruto. Los frutos de las palmeras silvestres no producen una cantidad óptima de aceites para competir comercialmente con especies genéticamente modificadas como la palma africana o el cocotero. La baja productividad de aceites es un limitante para la proyección comercial de las palmeras oleaginosas; sin embargo, al ser especies con una amplia distribución geográfica en el neotrópico y sujetas a uso por los pueblos amerindios, poseen una amplia variabilidad genética (de origen natural y antropogénico) no explorada. Parte de 
esta variabilidad está relacionada a las características morfológicas, anatómicas y bioquímicas del fruto, las cuales podrían ser utilizadas para el mejoramiento de la productividad y calidad de los aceites derivados. Balick (18) reporta que los indígenas poseen un amplio conocimiento de las diferentes formas -en sabor, contenido de aceites, coloración, facilidad de colección- de los frutos de O. bataua en la Amazonia. Por ejemplo, las formas híbridas $O$. bataua x $O$. ba$c a b a$, provenientes de Colombia y Venezuela, producen frutos excepcionalmente carnosos que podrían constituir un avance significativo al mejoramiento de la productividad de aceites provenientes del mesocarpo (32). Los frutos de la variedad $O$. bataua var. oligocarpa tienen un mayor porcentaje de contenido de aceite en el mesocarpo, así como una composición química con una mayor fracción de ácidos grasos saturados que la variedad bataua (3). Una fuerte variación en porcentajes de carbohidratos y aceites se reportan entre las variedades cultivadas de Bactris gasipaes (40). El conocimiento de la variabilidad del fruto a nivel infra-específico y de formas híbridas no ha sido estudiado; y potencialmente nuevas variantes con mayor porcentaje de mesocarpo, mayor productividad de aceites y mejores características de calidad en frutos permanecen escondidas en los bosques tropicales.

La productividad de los frutos de las palmeras silvestres no está limitado al volumen del mesocarpo, también depende de la capacidad individual de la palmera en producir múltiples infructescencias. Por ejemplo, bajo determinadas condiciones agronómicas, los individuos de $O$. bataua pueden incrementar su producción de infructescencias de 2 a 5 por año (18), lo que constituye un avance significativo al incremento de la productividad de la especie.

La investigación agronómica y el mejoramiento genético podrían favorecer a la domesticación y selección de caracteres agronómicamente importantes para las palmeras silvestres (productividad del fruto, lento crecimiento vertical, rápido desarrollo sexual). En el caso particular de la palma africana (Elaeis guineensis), las primeras plantaciones comerciales fueron establecidas en Sumatra en el año 1911. A partir de 1960, se desarrollaron clones más resistentes de enfermedades, de mayor producción y maduración favoreciendo el desarrollo comercial de esta especie (50). La domesticación de la palma africana cubre un periodo aproximado de 90 a 100 años para obtener individuos/clones de interés comercial de lento crecimiento vertical, alta productividad, resistente a enfermedades, entre otros caracteres agrícolas. Hoy en día, con el desarrollo tecnológico en ingeniería agronómica y genética, la domesticación de especies silvestres de palmeras podría llevarse a cabo en menos tiempo que el reportado para la palma africana. 
La conservación de la base genética de las poblaciones silvestres.- La variabilidad genotípica y fenotípica constituye la materia prima para la domesticación y el mejoramiento genético de las especies vegetales promisorias. Sin embargo, la conservación de la diversidad genética de las poblaciones silvestres de palmeras está amenazada por la transformación de bosques en tierras agropecuarias, la degradación del hábitat y el extractivismo destructivo $(51,52)$. Los remanentes de vegetación en el litoral ecuatoriano y en las estribaciones andinas occidentales albergan las últimas poblaciones de palmeras en esta región; si estos remanentes son transformados a tierras agrícolas, una parte importante de la base genética habrá desaparecido (53). La degradación del habitat (por efecto de borde, fragmentación, tala selectiva, etc.) altera la estructura poblacional y la regeneración natural de las poblaciones de palmeras $(19,54,55,56)$. En amplias áreas de pastizales y bosques secundarios de la provincia de Manabí, la estructura poblacional de Attalea colenda están formada casi exclusivamente por individuos adultos; la ausencia de regeneración natural refleja la poca viabilidad biológica de estas poblaciones a futuro. La cosecha de frutos de palmeras con técnicas que involucra la tala de los individuos ha provocado la disminución progresiva de las poblaciones silvestres de palmeras en la Amazonía y la preocupación sobre su conservación a lar- go plazo (57). Durante los periodos de fructificación de Oenocarpus bataua, cientos (o aún miles) de individuos adultos son talados para la obtención de sus frutos en la Amazonía Peruana, preferentemente en las cercanías de los centros poblados. El impacto del extractivismo destructivo en la resiliencia de las poblaciones no ha sido evaluado en detalle; sin embargo, este tipo de actividad genera la pérdida sistemática de la diversidad genética local (52). Se estima que en la región de Loreto (Amazonía Peruana) se cortan 1000 palmeras femeninas de $M$. flexuosa mensualmente para la obtención de sus frutos (58). Frente a los factores que amenazan la integridad genética de las poblaciones de palmeras in situ es prioritario la implementación de bancos de germoplasma (semillas y $\mathrm{ADN}$ ), tanto a nivel nacional como regional (Colombia, Ecuador, Perú y Bolivia). La conservación de las variedades locales en bancos de germoplasma juegan un rol importante como proveedores de alelos para el mejoramiento genético y conservación de las especies. En base a la información disponible, las accesiones de palmeras nativas depositadas en bancos de germoplasma a nivel regional se limitan a colecciones de Bactris gasipaes (40) y en algunos casos Elaeis oleifera.

Un ejemplo de la importancia de la conservación de la diversidad genética constituyen los programas de mejoramiento genético de la palma africana. 
Híbridos inter-específicos (Elaeis guineensis x E. oleifera) han sido generados para mejorar las características agronómicas y la resistencia de enfermedades a través de introgresiones de rasgos importantes de E. oleifera, en particular la resistencia contra la pudrición (59).

\section{Reservas extractivistas de palme-} ras.- Las reservas extractivistas son un innovativo modelo de desarrollo en donde se fusionan la protección ambiental con el manejo sustentable bajo el ideal que supone el aprovechamiento del bosque sin destruirlo. Este modelo de aprovechamiento de los recursos biológicos podría ser experimentado para algunas especies de palmeras con amplias poblaciones silvestres en las regiones tropicales. Para el año 2000, Brazil tenía 16 reservas extractivistas con un área de 34079 kilómetros cuadrados (60). En un inicio, estas reservas fueron creadas para regular el aprovechamiento del látex de Hevea brasilensis, y posteriormente nuevos productos del bosque, como los frutos de palmeras, fueron incluidos en el modelo de extractivismo. Las reservas extractivistas han dado resultados positivos reduciendo la presión de deforestación sobre del bosque; sin embargo, varias preguntas necesitan ser exploradas previa su aplicación con las palmeras: (a) ¿Cuáles son los efectos del extractivismo de frutos en la diversidad y dinámica del bosque considera- do que los frutos de palmeras son una importante fuente de alimentos para la fauna local? Estudios llevados a cabo con E. oleracea reportan que modelos de cosecha con remoción de más del 75 $\%$ de frutos generan una reducción en la diversidad de aves frugívoras, con consecuencias en la dinámica del bosque (61). (b) ¿Los bosques tropicales producen la cantidad de frutos necesaria por área para procesos de comercialización a mediana escala?. Datos provenientes de la Amazonia Peruana revelan que la producción de frutos es menor a lo que asumen los conservacionistas (62). (c) ¿La productividad de frutos es estable durante el año?. La productividad del bosque es variable a nivel espacial y temporal. Los bosques altamente diversos de la Amazonia (tierra firme) producen significantemente menos frutos que bosques aluviales y pantanos; y esta productividad esta relacionada a la fenología de las especies (62). (d) ¿Cuál es el método adecuado para cosechar frutos de palmeras arborescentes? .

Los modelos extractivistas pueden ser exitosos para especies que crece en (a) extensas áreas de la Amazonía, (b) sobre suelos no aptos para la agricultura (pantanos), y (c) en altas densidades. Bajo estas características modelos de extractivismo pueden ser opciones viables para especies como Mauritia flexuosa y Oenocarpus bataua bajo un modelo de manejo sustentable (63). 


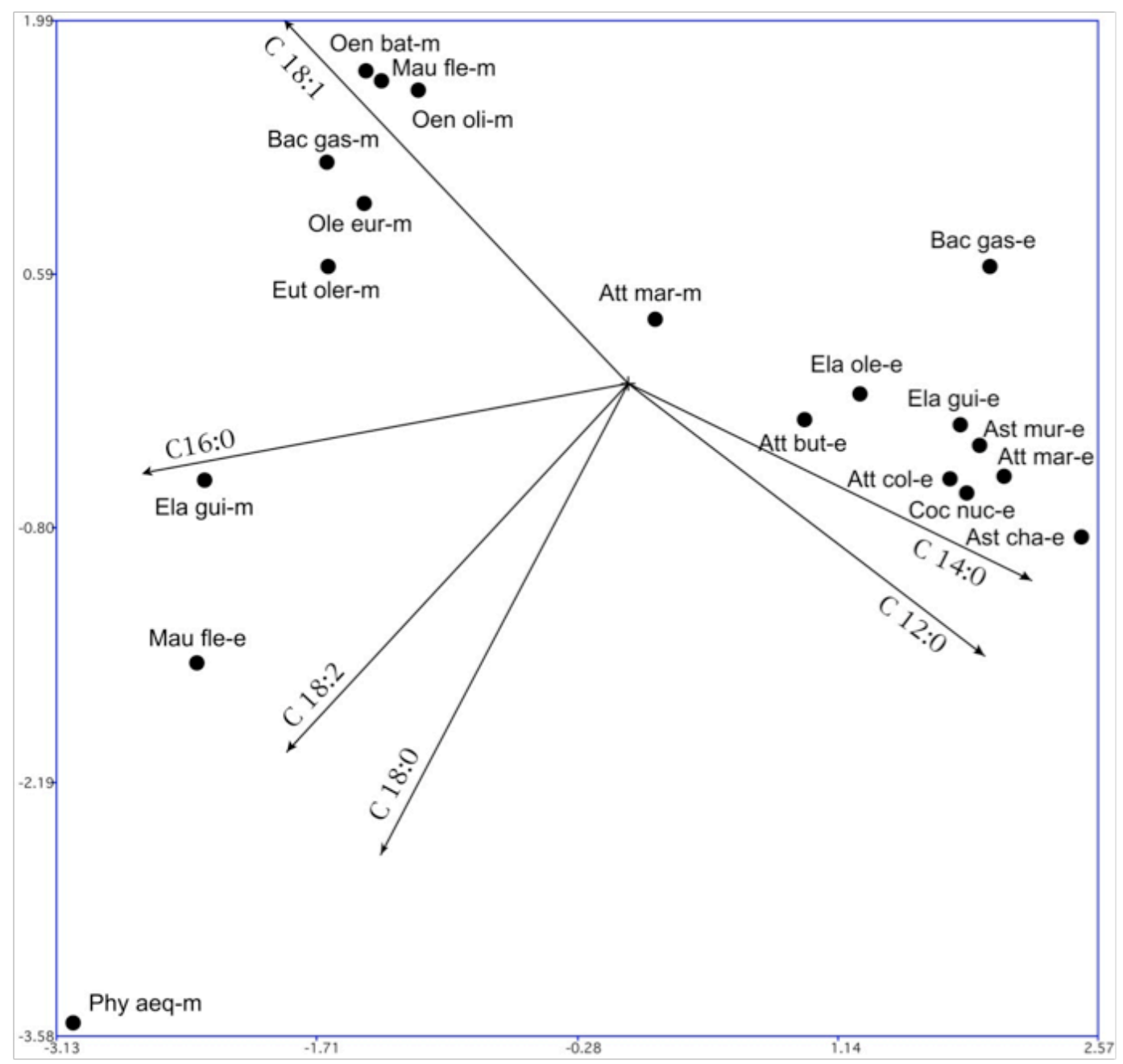

Figura 1. Posición relativa de 19 muestras de aceites derivados de palmeras (basado en información bibliográfica) en un espacio definido por dos componentes principales (PCA). Los componentes principales fueron construidos con la información de seis ácidos grasos como descriptores (C12:0; C14:0; C16:0; C18:0; C18:1; C18:2). Los círculos sólidos representan las muestras incluidas en el análisis $(\mathrm{n}=19)$. Las flechas representan el peso de cada eigenvector en los dos primeros ejes. Los dos primeros componentes representan el $86.11 \%$ de varianza total. Abreviaturas: Ast cha-e: Astrocaryum chambira/endospermo; Phy aeq-m: Phytelephas aequatorialis/mesocarpo; Mau fle-e: Mauritia flexuosa/endospermo; Ela gui-m: Elaeis guineensis/mesocarpo; Coc nuc-e: Cocos nucifera/endospermo; Att col-e: Attalea colenda/endospermo; Att mar-e: Attalea maripa/endospermo; Ast mur-e: Astrocaryum murumuru/endospermo; Ela gui-e: Elaeis guianeensis/ endospermo; Atta but-e: Attalea butyracea/endospermo; Ela ole-e: Elaeis oleifera/endospermo; Att mar-m: Attalea maripa/mesocarpo; Eut ole-m: Euterpe oleracea/mesocarpo; Ole eur-m: Olea europaea/mesocarpo; Bac gas-m: Bactris gasipaes/mesocarpo; Bac gas-e: Bactris gasipaes/endosperma; Oen oli-m: Oenocarpus batanavariedad oligocarpa/mesocarpo; Oen bat-m: Oenocarpus batauavariedad bataua/mesocarpo; Mau flem: Mauritia flexuosa/mesocarp 


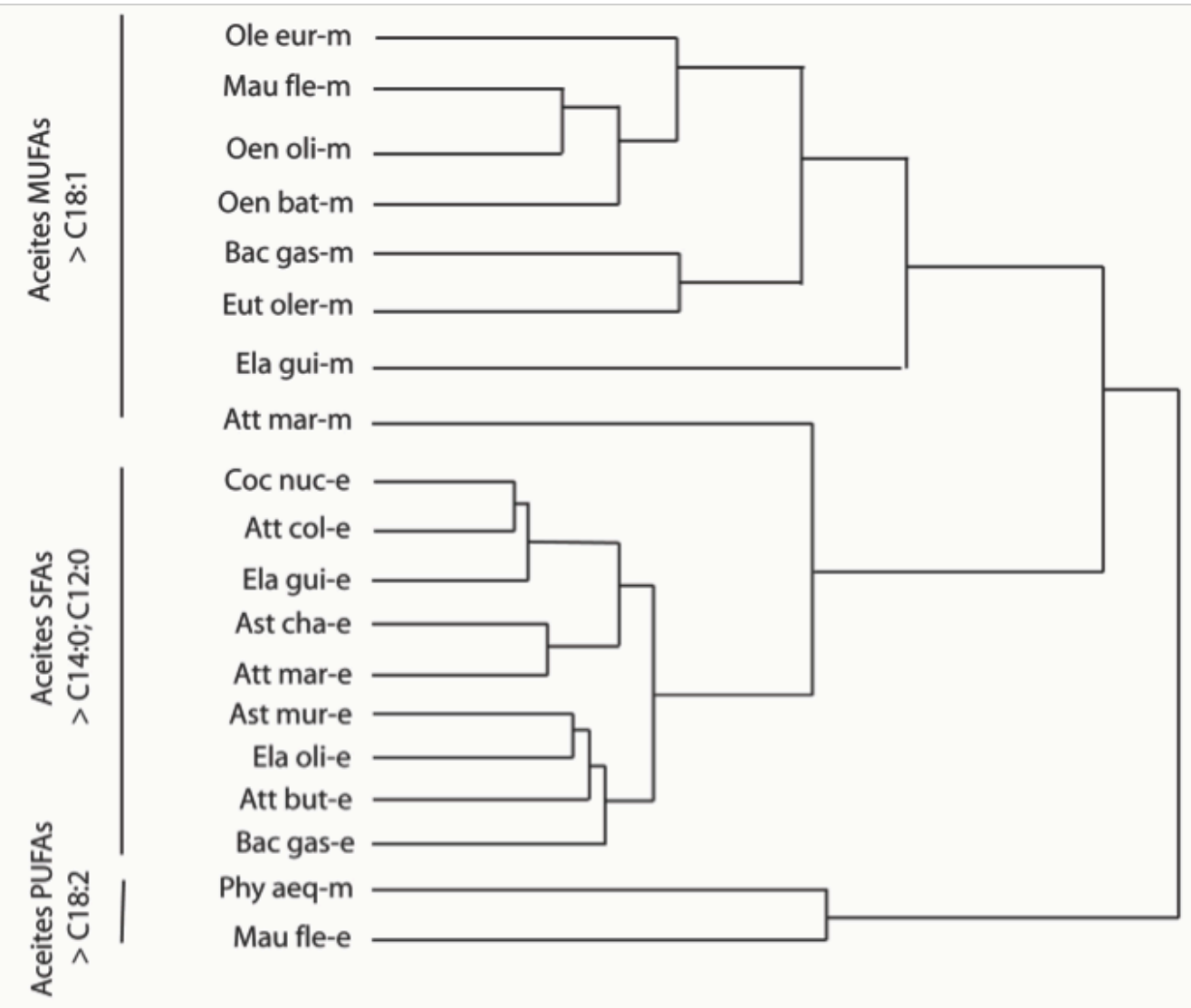

Figura 2. Análisis UPGMA de 19 muestras de aceites derivados de palmeras (información bibliográfica) con la información de seis ácidos grasos (C12:0; C14:0; C16:0; C18:0; C18:1; C18:2). Abreviaturas: ver figura 1

\section{CONCLUSIONES}

El concepto de especies promisorias fue difundido en Latinoamérica a partir de la década de los 70s; sin embargo, escasos avances se han realizado en la región para el manejo comercial e industrialización de las poblaciones de palmeras oleaginosas. La actual coyuntura internacional en busca de nuevas fuentes de aceites saludables para el hombre y de biocombustibles favorece el impulso de la investigación científica sobre palmeras y aceites. Dentro de este contexto, es indispensable que el Estado, la universidad y la empresa privada desarrollen, en conjunto, líneas de investigación dirigidas hacia el estudio de la (a) variabilidad bioquímica, (b) la adaptación agronómica, y (c) la conservación y manejo de la diversidad genética de las fuentes de 


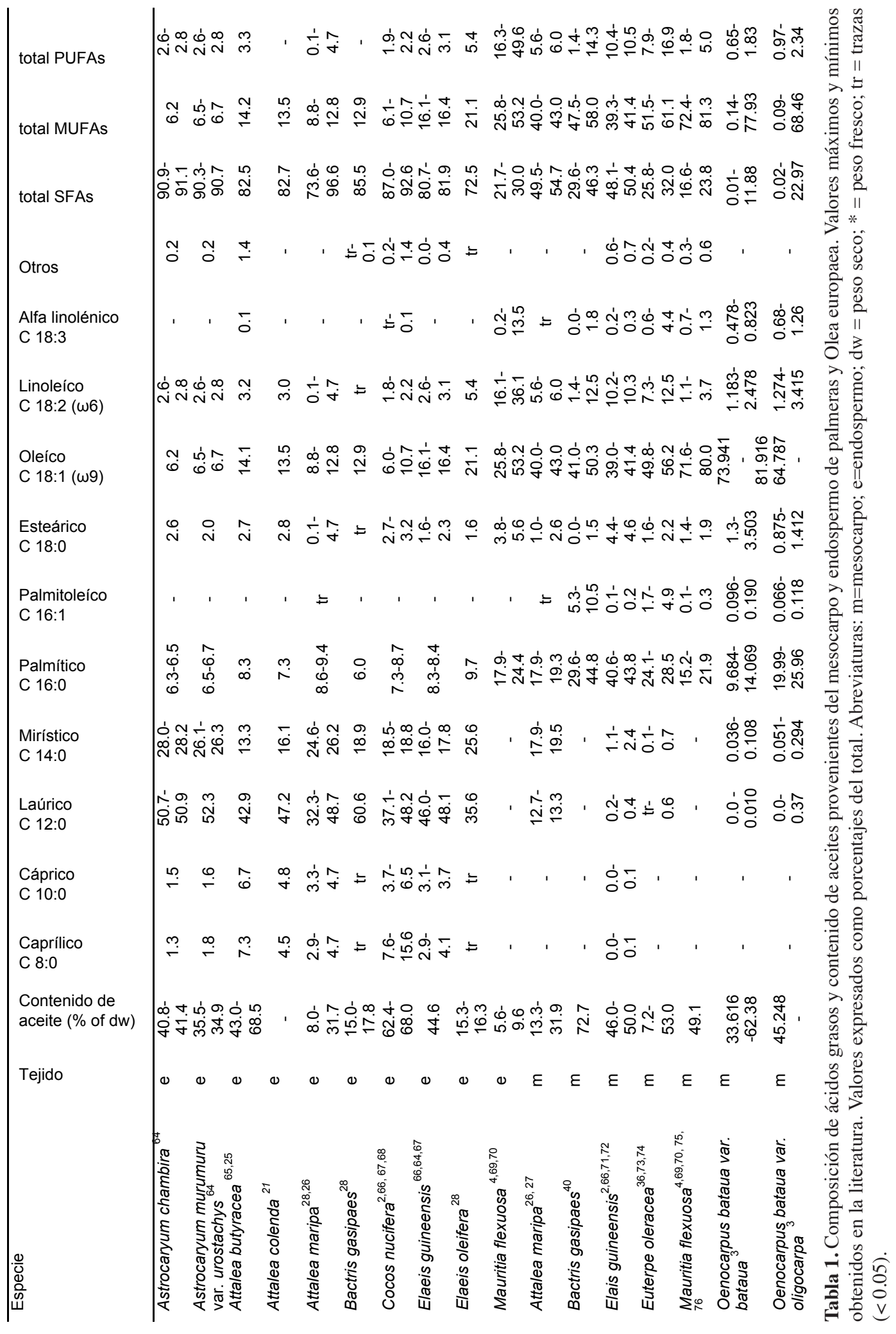


oleaginosas del Ecuador; información indispensable para emprender procesos de investigación a largo plazo como la domesticación y mejoramiento genético. La alta diversidad de palmeras del Ecuador ofrece un escenario único para el desarrollo de investigación en oleaginosas; y es probable que nuestros bosques tropicales alberguen a las futuras especies comerciales que producirán nuestros aceites comestibles e industriales.

\section{AGRADECIMIENTOS}

El presente estudio fue financiado por la Unión Europea-Séptimo Programa Marco "Palm Harvest Impact in Tropical Forest" (FP7-PALMS, grant \# 212631). Agradecemos a dos revisores anónimos por sus comentarios al manuscrito.

\section{REFERENCIAS \\ BIBLIOGRÁFICAS}

1. FAO. 2010. Fats and fatty acids in Human Nutrition. Report of an expert consultation. FAO/Food and Nutrition Paper 91.

2. DUBOIS, V.; BRETON, S.; LINDER, M.; FANNI, J. \& PARMENTIERA, M. 2007. Fatty acid profiles of 80 vegetable oils with regard to their nutritional potential. European Journal of Lipid Science and Technology. 109: 710-732.

3. MONTÚFAR, R. 2007. Structure génétique, biochemique, morpho- logique et écologique de Oenocarpus bataua Mart. (Arecaceae): perspectives pour la valorisation durable d'une ressource forestière néotropicale. Tesis doctoral. Ecole Nationale Supérieure Agronomique de Montpellier, Francia.

4. JACOBO, F. Q.; ROJAS, M. A.; REYES, G. I.; PINO, E. L. \& CHAGMAN, G. P. 2009. Caracterización de aceites, tortas y harinas de frutos de Ungurahui (Jessenia polycarpa) y Aguaje (Mauritia flexuosa L.) de la Amazonía Peruana. Revista de la Sociedad Química del Perú. 75(2): 243-253.

5. KOZIOL, M. J. \& BORGTOFT PEDERSEN, H. 1993. Phytelephas aequatorialis (Arecaceae) in human and animal nutrition. Economic Botany. 47(4): 401-407.

6. DAMUDE, H. G. \& KINNEY, A. 2008. Enhancing plant seed oils for human nutrition. Plant Physiology. 147: 962-968.

7. KROMHOUT, D.; MENOTTI, A.; BLOEMBERG, B.; ARAVANIS, C.; BLACKBURN, H.; BUZINA, R.; DONTAS, A. S.; FIDANZA, F.; GIAIPAOLI, S.; JANSEN, A.; KARVONEN, M.; KATAN, M.; NISSINEN, A.; NEDELJKOVIC, S.; PEKKANEN, J.; PEKKARINEN, M.; PUNSAR, S.; RASANEN, L.; SIMIC, B. \& TOSHIMA H. 1995. Dietary Saturated and 
trans Fatty Acids and Cholesterol and 25-Year Mortality from Coronary Heart Disease: The Seven Countries Study. Preventive Medicine. 24(3): 308-315.

8. FAO/OMS. 1993. Grasas y aceites en la nutrición humana. Consulta FAO/OMS, Roma 19-26 octubre 1993.

9. BANCO MUNDIAL. 2007. Insuficiencia nutricional en Ecuador. Quito, Ecuador. Página de Internet: http://ecuador.nutrinet.org/ecuador/ situacion-nutricional. Consultada 1-junio-2011.

10. FAOSTAT. 2010. Organización de las naciones Unidas para la agricultura y alimentación. Página de Internet: http://faostat.fao.org/ site/565/default.aspx. Consultada 1-junio-2011.

11. PINTAUD, J. C.; GALEANO, G.; BALSLEV, H.; BERNAL, R.; BORCHSENIUS, F.; FERREIRA, E.; DE GRANVILLE, J. J.; MEJIA, K.; MILLAN, B.; MORAES, M.; NOBLICK, L.; STAUFFER, F. \& KAHN, F. 2008. Las palmeras de América del Sur: diversidad, distribución e historia evolutiva. Revista Peruana de Biología. 15(1):7-29.

12. DE LA TORRE, L.; NAVARRETE, H.; MURIEL, P.; MACÍA, M. \& BALSLEV, H. (eds.). 2008. Enciclopedia de las plantas útiles del Ecuador. Herbario QCA de la Es- cuela de Ciencias Biológicas de la Pontificia Universidad Católica del Ecuador \& Herbario AAU del Departamento de Ciencias Biológicas de la Universidad de Aarhus. Quito $\&$ Aarhus.

13. BARFOD, A. \& KVIST, L. P. 1996. Comparative Ethnobotanical Studies of the Amerindian groups in Coastal Ecuador. Biological Papers 46. The Royal Danish Academy of Science and Letters, Copenhagen.

14. BARFOD, A. S. \& BALSLEV, H. 1998. The use of the Palms by the Cayapas and Coaiqueres on the Coastal Plain of Ecuador. Principes. 32:29-41.

15. CERON, C. E. \& MONTALVO, C. 1998. Etnobotánica de los Huaorani de Quehueiri-Ono, Napo-Ecuador. Primera edición. Abya-Yala, Quito.

16. LLERAS, E. \& CORADIN, L. 1988. Native Neotropical oil Palms: State of the Art and Perspectives for Latin American. Advances in Economic Botany. 6: 201-213.

17. BALICK, M. J. 1984. Ethnobotany of Palms in the Neotropics. Advances in Economic Botany. 1: 9-23.

18. FAO. 1988. Jessenia and Oenocarpus: neotropical oil Palms worthy of domestication. FAO Plant Production and Protection Paper 88, Rome.

19. BORCHSENIUS, F.; BORGTOFT PEDERSEN, H. \& BALSLEV, H. 1998. Manual to the Palms of Ecua- 
dor. AAU reports 37. Department of Systematic Botany, University of Aarhus, Denmark.

20. BALSLEV, H. \& HENDERSON, A. 1987. The Identity of Ynesa colenda (Palmae). Brittonia. 39:1-6.

21. BLICHER-MATHIESEN, U. \& BALSLEV, H. 1990. Attalea colen$d a$, a potencial lauric oil resource. Economic Botany. 44(3): 360-368.

22. ACOSTA SOLIS, M. 1960. Palmas económicas del Noroccidente Ecuatoriano. Editorial Ecuador, Quito.

23. FEIL, J. P. 1996. Fruit production of Attalea colenda (Arecaceae) in Coastal Ecuador- an alternative oil resource? Economic Botany. 50(3): 300-309.

24. BERNAL, R.; GALEANO, G.; GARCÍA, N.; OLIVARES, I. L. \& COCOMÁ, C. 2010. Uses and commercial prospects for the Wine Palm Attalea butyracea, in Colombia. Ethnobotany Research \& Aplications. 8: 255-268.

25. DEVIA, J. E.; LÓPEZ, A. \& SALDARRIAGA, O. L. 2002. Productos promisorios del fruto de la palma de vino. Rev. Univ. Eafit, Colombia 126. 67-80.

26. BEREAU, D.; BENJELLOUNMLAYAH, B. \& DELMAS, M. 2001. Maximiliana maripa Drude Mesocarp and Kernel Oils: Fatty Acid and Total Tocopherol Compositions. Journal of the American Oil Chemists`Society. 78: 213-214.
27. CORZO, O.; BRACHO, N.; VÁSQUEZ, A. \& A. PEREIRA. 2010. Determination of suitable thin layer model for drying of coroba slices (Attalea maripa) at different air temperatures and velocities. Journal of Food Processing and Preservation. 34: 587-598.

28. BEREAU, D.; BENJELLOUNMLAYAH, B.; BANOUB, J. \& BRAVO, R. 2003. FA and Unsaponifiable Composition of Five Amazonian Palm Kernel Oils. Journal of the American Oil Chemists' Society. 80(1): 49-53.

29. HENDERSON, A.; GALEANO, G. \& BERNAL, R. 1995. Field Guide to the Palms of the Américas. Princeton University Press, New Jersey.

30. ANDERSON, A. \& OVERAL, W. 1988. Pollination ecology of a Forest-Dominant palm (Orbignya phalerata Mart.) in Northern Brazil. Biotropica. 20:192-205.

31. MAY, P. H.; ANDERSON, A.; FRAZAO, J. M. \& BALICK, M. 1985. Babassu palm in the agroforestry systems in Brazil’s Mid-North region. Agroforestry Systems, 3. 275-295.

32. BALICK, M. J. 1981. Une huile comestible de haute qualité en provenance des espèces Jessenia et Oenocarpus: un complexe de palmiers natifs de la Vallée de 1'Amazone. Oléagineux. 36: 319-326. 
33. BALICK, M. J. \& GERSHOFF, S. N. 1981. Nutritional evaluation of the Jessenia bataua palm: Source of high quality protein and oil from Tropical America. Economic Botany. 35: 261-271.

34. BALICK, M. J. 1986. Systematics and Economic Botany of the Oenocarpus-Jessenia (Palmae) complex. Advances in Economic Botany 3. $1-140$.

35. BORGTOFT-PEDERSEN, H. \& BALSLEV, H. 1993. Palmas útiles. Especies ecuatorianas para agroforestería y extractivismo. Ediciones Abya Yala, Quito.

36. MONTÚFAR, R.; LAFFARGUE, A.; PINTAUD, J-C.; HAMON, S.; AVALLONE, S. \& DUSSERT, S. 2010. Oenocarpus bataua Mart. (Arecaceae): Rediscovering a Source of High Oleic Vegetable Oil from Amazonia. Journal of the American Oil Chemists' Society. 87:167-172.

37. SALAZAR, E.; BELÉN, D.; JIMÉNEZ, N. \& PINO, K. 2004. Características físico químicas del aceite de la semilla de la coroba (Jessenia polycarpa Karst). Grasas y Aceites. 55(4): 423-427.

38. DIAZ, J. A. \& ÁVILA, L. M. 2002. Sondeo del mercado mundial de Aceite de Seje (Oenocarpus bataua) Instituto de Investigación de Recursos Biológicos Alexander von Humboldt, Bogotá, Colombia. 18 pp.
39. MILLER, C. 2002. Fruit production of the Unguahua palm (Oenocarpus bataua subsp. bataua, Arecaceae) in an indigenous managed reserve. Economic Botany. 56(2): 165-176.

40. MORA-URPÍ, J.; WEBER, J. C. \& CLEMENT, C. R. 1997. Peach palm. Bactris gasipaes Kunth. Promoting the conservation and use of underutilized and neglected crops. 20. Institute of Plant Genetics and Crop Plant Research, Gatersleben/ International Plant Genetic Resources Institute, Rome, Italy.

41. CORPEI, 2009. Perfiles de Producto. Perfiles del Palmito. Centro de Información e Inteligencia Comercial (CICO). CORPEI.

42. HEISER, C. 1990. New perspectives on the origin and evolution of new world domesticated plants: Summary. Economic Botany. 44: 111-116.

43. COUVREUR, T.; BILlOTE, N.; RISTERUCCI, M.; LARA, C.; VIGOUROUX, Y.; LUDEÑA, B.; PHAM, J-L. \& PINTAUD, J-C. 2006. Close genetic proximity between cultivated and wild Bactris gasipaes Kunth revealed by microsatellite markers in western Ecuador. Genetic Resources and crop Evolution. 53:1361-1373.

44. HERNÁNDEZ-UGALDE, J. A.; MORA-URPI, J. \& ROCHA NU- 
ÑEZ, O. 2008. Diversidad genética y relaciones de parentesco de las poblaciones silvestres y cultivadas de pejibaye (Bactris gasipaes, Palmae), utilizando marcadores microsatelitales. Revista de Biología Tropical. 56(1): 217-245.

45. BARCELOS, E.; AMBLARD, P.; BERTHAUD, J. \& SEGUIN, M. 2002. Genetic diversity and relationship in American and African oil palm as revealed by RFLP and AFLP molecular markers. Pesquisa Agropecuária Brasileira. 37(8): 1105-1114

46. MORETZSOHN, M. C.; FERREIRA, M. A.; AMARAL, Z. P. S.; COELHO, P. J. A.; GRATTAPAGLIA, D. \& FERREIRA, M. E. 2002. Genetic diversity of Brazilian oil palm (Elaeis oleifera H.B.K.) germplasm collected in the Amazon Forest. Euphytica. 124: 35-45.

47. LEGENDRE, P. \& LEGENDRE L. 1998. Numerical Ecology. Developments in environmental Modeling 20. Second Edition.

48. VERGARA, L. K. 2002. Demografía de Ceroxylum alpinum en bosques relictuales Del Valle de Cocora, Salento (Quindio). Tesis de grado. Universidad Nacional de Colombia.

49. FORERO, L. E. 1983. Anotaciones sobre bibliografia seleccionada del complejo Jessenia-Oenocarpus (Palmae). Cespedesia. 12: 21-49.
50. HENDERSON, J. \& OSBORNE D. 2000. The oil palm in all our lives: how this came about. Endeavour. 24(2): 63-68.

51. CONWAY, W. 2009. Conservation genetics and the extinction crisis. En: Amato et al. eds. Conservation Genetics in the age of genomics. Columbia University Press.

52. MONTÚFAR, R.; ANTHELME, F.; PINTAUD, J-C. \& BALSLEV, H. 2011. Effects of disturbance on resilience of tropical American palm populations and communities. En prensa, The Botanical Review.

53. MOSANDL, R.; GUNTER, S.; STIMM, B. \& WEBER, M. 2008. Ecuador suffers the highest deforestation rate in South America. En: Beck et al. eds. Gradients in a Tropical Mountain Ecosystem of Ecuador. Ecological Studies, 198. 37-40.

54. ANTHELME, F.; LINCANGO, J.; GULLY, C.; DUARTE, N. \& MONTÚFAR, R. 2011. How anthropogenic disturbances affect the resilience of a keystone palm tree in the threatened Andean cloud forest ?. Biological Conservation. 144: 1059-1067.

55. AGUIRRE, A. \& DIRZO, R. 2008. Effects of fragmentation on pollinator abundance and fruit set of an abundant understory palm in a Mexican tropical forest. Biological Conservation. 141: 375-384. 
56. GALETTI, M.; DONATTI, C. I.; PIRES, A. S.; GUIMARÃES, P. R. JR. \& JORDANO, P. 2006. Seed survival and dispersal of an endemic Atlantic forest palm: the combined effects of defaunation and forest fragmentation. Botanical Journal of the Linnean Society. 151: 141-149.

57. KAHN, F. 1988. Ecology of Economically Important Palms in Peruvian Amazonia. Advances in Economic Botany. 6: 42-49.

58. ROJAS RUIZ, R,; RUIZ PANDURO, G.; RAMÍREZ MELÉNDEZ, P.; SALAZAR JARAMA, C.; RENGIFO SIAS, C.; LLERENA FLORES, CH.; MARÍN RÍOS, C.; TORRES NORIEGA, D.; OJANAMA VÁSQUEZ, J.; SILVANO ALVÁN, W.; MUÑOZ ISUIZA, V.; LUQUE SALINAS, H.; VELA GONZA, N.; CASTILLO FASABI, N.; SOLIGNAC RUIZ, J.; R. LÓPEZ DE OLIVEIRA, V.; PANDURO RUIZ, F. 2001. Comercialización de masa y «fruto verde» de aguaje (Mauritia flexuosa L.f.) en Iquitos (PERÚ). Folia Amazónica. 12(1-2) - 2001.

59. AMBLANRD, P.; BILLOTTE, N.; COCHARD, B.; DURAND-GASSELIN, T.; JACQUEMARD, J. C,; LOUISE, C.; NOUY, B.; POTIER, F. 2004. Elaeis guineensis and Elaeis oleifera oil palm breeding at CiradCP. Palmas-No. Especial, 2. 306-310.
60. RUIZ-PÉREZ, M.; ALMEIDA, M.; DEWI, S.; LOZANO COSTA, E.; CIAVATTA PANTOJA, M.; PUNTODEWO, A.; DE ARRUDA POSTIGO, A. \& GOULART DE ANDRADE, A. 2005. Conservation and Development in Amazonian Extractive Reserves: The Case of Alto Juruá. Ambio. 34(3): 218-223.

61. MOEGENBURG, S. M. \& LEVEY, D. J. 2002. Prospects for conserving biodiversity in Amazonian extractive reserves. Ecology Letter 5(3): 320-324.

62. PHILLIPS, O. 1993. The potencial for harvesting fruits in tropical rainforest: new data from Amazonian Perú. Biodiversity and Conservation 2. 18-38.

63. PETERS, C.; BALICK, M. J.; KAHN, F. \& ANDERSON, A. B. 1989. Oligarchic forest of economic plants: Utilization and Conservation of an important Tropical resource. Conservation Biology, 3. 342-349.

64. SHUKLA, V. K. S. \& HOLM JENSEN, O. 1996. Fatty acid composition and tocopherol content of Amazonian palm oils. Journal of Food Lipids. 3: 149-154.

65. MADRE TIERRA AMAZONIA. 2010. Ficha técnica Aceite de Pa1la. Madre Tierra Amazonia S.R.L./ IPHAE Riberalta, Bolivia. 
66. LÍSA, M. \& HOLČAPEK, M. 2008. Triacylglycerols profiling in plant oils important in food industry, dietetics and cosmetics using high-performance liquid chromatography-atmospheric pressure chemical ionization mass spectrometry. Journal of Chromatography A, 1198-1199: 115-130.

67. GUNSTONE, F. D. 2002. Production and trade of vegetable oils. En: Gunstone, F. D. ed. Vegetable oils in food and technology: Composition, Propierties and uses. Blackwell Publishing Ltd. Pp. 1-17.

68. PANTZARIS, T. P. \& BASIRON, Y. 2002. The lauric (coconut and palmkernel) oils. En: Gunstone, F. D., ed. Vegetable oils in food and technology: Composition, Properties and Uses. Blackwell Publishing Ltd. Pp. 157-202.

69. MADRE TIERRA AMAZONIA. 2010. Ficha técnica Aceite de Palma Real. Madre Tierra Amazonia S.R.L./IPHAE Riberalta, Bolivia.

70. VÁSQUEZ-OCMÍN, P. G.; FREITAS ALVARADO, L.; SOTERO SOLÍS, V.; PAVÁN TORRES, R. \& MANCINI-FILHO, J. 2010. Chemical characterization and oxidative stability of the oils from three morphotypes of Mauritia flexuosa L.f, from the Peruvian Amazon. Grasas y aceites. 61(4): 390-397.
71. FRANKE, S.; FRÖHLICH, K.; WERNER, S.; GRAF, T.; BÖHM, V. \& SCHÖNE, F. 2008. Carotinoide, Vitamin E und weitere qualitätsrelevante Bestandteile der Saat, des Presskuchens und des Öles von Raps, Sonnenblume, Lein und Distel. UFOP: Bericht Carotenoide: 1-31. Página internet: http://www. ufop.de/downloads/Bericht_Carotinoide_110609\%281\%29.pdf. Consultada 20-octubre-2010.

72. YUSOFF, S. 2006. Renewable energy from palm oil - innovation on effective utilization of waste. Journal of Cleaner Production. 14: 87-93.

73. SCHAUSS, A. G.; WU, X.; PRIOR, R. L.; OU, B.; PATEL, D.; HUANG, D. \& KABABICK, J. P. 2006. Phytochemical and Nutrient Composition of the Freeze-Dried Amazonian Palm Berry, Euterpe oleraceae Mart. (Acai). Journal of Agricultural and Food Chemistry. 54: 8598-8603.

74. DO NASCIMENTO, R. J. S.; COURI, S.; ANTONIASSI, R. \& FREITAS, S. P. 2008. Composição em ácidos graxos do óleo da polpa de Açaí extraído com enzimas e com hexano. Revista Brasileira de Fruticultura, Jaboticabal - SP, Vol. 30(2): 498-502.

75. ALBUQUERQUE, M. L. S.; GUEDES, I.; ALCANTARA JR. 
P.; MOREIRA, S. G. C.; BARBOSA N. M.; CORREAC D. S. \& ZILIOC, S. C. 2005. Characterization of Buriti (Mauritia flexuosa L.) Oil by Absorption and Emission Spectroscopies. Journal of the Brazilian Chemical Society, Vol. 16(6A): 1113-1117.

76. SANTOS, L. M. P. 2005. Nutritional and ecological aspects of buriti or aguaje (Mauritia flexuosa Linnaeus filius): A carotene-rich palm fruit from Latin America. Ecology of Food and Nutrition. 44(5): 345-358.
77. LAVEE, S. \& WODNER, M. 2004. The effect of yield, harvest time and fruit size on the oil content in fruits of irrigated olive trees (Olea europaea), cvs. Barnea and Manzanillo. Scientia Horticulturae. 99: 267-277.. 